\title{
Mathematical Modeling of the Electrophysical Properties of a Layered Nanocomposite Based on Silicon with an Ordered Structure
}

\author{
Sergey Korchagin ${ }^{1}(0)$, Ekaterina Romanova ${ }^{1, *}{ }^{\oplus}$, Denis Serdechnyy ${ }^{2}$, Petr Nikitin $^{3} \oplus$, Vil Baiburin ${ }^{4}$ \\ and Yerbol Yerbayev ${ }^{5}$ \\ 1 Department of Data Analysis and Machine Learning, \\ Financial University under the Government of Russian Federation, 4th Veshnyakovsky pr. 4, \\ 111395 Moscow, Russia; SAKorchagin@fa.ru \\ 2 Department of Innovation Management, State University of Management, Ryazansky pr. 99, \\ 109542 Moscow, Russia; dv_serdechnyj@guu.ru \\ 3 Department of Applied Informatics, Russian State Agrarian University-Moscow Timiryazev \\ Agricultural Academy, Timiryazevskaya Street 49, 127550 Moscow, Russia; petrvlni@rambler.ru \\ 4 Department of Information Security of Automated Systems, \\ Yuri Gagarin State Technical University of Saratov, Polytechnic Street 77, 410054 Saratov, Russia; \\ baiburinvb@rambler.ru \\ 5 Higher School of Mechanical Engineering, Zhangir Khan West Kazakhstan Agrarian-Technical University, \\ Zhangir Khan Street 51, Uralsk 090009, Kazakhstan; erbol.erbaev@mail.ru \\ * Correspondence: ekvromanova@fa.ru or EkVRomanova@fa.ru; Tel.: +7-906-757-32-54
}

check for updates

Citation: Korchagin, S.; Romanova, E.; Serdechnyy, D.; Nikitin, P.; Baiburin, V.; Yerbayev, Y.

Mathematical Modeling of the Electrophysical Properties of a Layered Nanocomposite Based on Silicon with an Ordered Structure. Mathematics 2021, 9, 3167. https:// doi.org/10.3390/math9243167

Academic Editor: Amir Mosavi

Received: 6 November 2021

Accepted: 7 December 2021

Published: 9 December 2021

Publisher's Note: MDPI stays neutral with regard to jurisdictional claims in published maps and institutional affiliations.

Copyright: (C) 2021 by the authors Licensee MDPI, Basel, Switzerland. This article is an open access article distributed under the terms and conditions of the Creative Commons Attribution (CC BY) license (https:/ / creativecommons.org/licenses/by/ $4.0 /)$.

\begin{abstract}
The authors carried out modeling of the electrophysical properties of composite media. The frequency dependences of the dielectric constant on the type of inclusions in the composite are investigated. On the basis of the nanocomposite considered in the work, based on $\mathrm{Si}, \mathrm{B}$, and $\mathrm{SiO}_{2}$, the authors model a reflecting screen, the lattice elements of which have a layered hierarchically constructed structure similar to a fractal formation. The influence of the level of fractality on the optical properties of the object was also investigated, and it was found that the proposed structure makes it possible to increase the operating frequency range of the reflecting screen and the efficiency, in comparison with reflecting screens that have a lattice of traditional structure. The results obtained can be of practical interest for broadband and nonlinear radar devices, localization devices and mobile objects, microelectronics, as well as intelligent applications in the field of information security.
\end{abstract}

Keywords: composite materials; fractional model; mathematical modeling; fractals; nonlinear dynamics; reflective screen; optical properties

\section{Introduction}

The increased interest of scientists, researchers, and engineers in modern composite materials is based, first of all, on a wide range of their physical and chemical properties in relation to various areas of human activity [1,2]. The prospect of using composite materials is due to many factors, the most important of which are the availability of raw materials [3] and multifunctionality [4]. Thanks to composites, the following applied problems are solved: Obtaining materials with high corrosion resistance [5], development of materials with high radioactive resistance [3], creation of new biologically compatible materials [6], synthesis of safe and environmentally friendly materials [7], microminiaturization of devices using composite media [8].

The uniqueness of composite materials lies in the fact that it is possible to design in advance the structure of the material in such a way as to give the product from it the properties necessary for solving a specific applied problem $[9,10]$. Material design can be one of the key factors affecting its functional properties [11]. Therefore, the determination of 
the electrodynamic properties of composite media depending on the design of the material is an urgent task.

Reflective screens for passive radar reflectors and reflectors are used as elements of innovative antenna designs [12], broadband and nonlinear radar devices [13], means for localizing mobile objects [14], selective and absorbing materials [15].

There are a large number of reflective screens made, for example, in the form of a paraboloid of revolution or a solid metal plate [16]. For such reflective screens, the following disadvantages can be distinguished-they are large mass and high aerodynamic resistance. Reflective screens made in the form of a thin conductive perforated plate [17], or in the form of a system of parallel thin metal plates, the planes of which are parallel to the direction of wave propagation and perpendicular to the electric field vector [18], have a lower mass. However, the disadvantage of these screens is the leakage through them of part of the power of the electromagnetic wave. The use of a reflective screen design containing a lattice of metal conductors makes it possible to further reduce the weight. However, it was found in $[19,20]$ that with an increase in the radiation frequency, a part of the power leaks out, which leads to a decrease in the reflection coefficient.

In this paper, we study the frequency dependences of the complex dielectric permeability of composite media consisting of a matrix with inclusions in the form of spheres and cylinders using the selection, improvement, and development of combined mathematical models of composite structures. As an example of practical application, the developed mathematical model of a reflecting screen of a fractal structure is presented. Currently, the following types of screens are known: A screen for reflector antennas, made in the form paraboloid of revolution made of solid metal [17], a reflecting screen made in the form of a plate of solid metal [21]. A common disadvantage of these reflective screens is their large mass since they are made of solid metal. In addition, they have great aerodynamic resistance. These flaws were not found in reflective screens made in the form of one thin conductive perforated plate [22], or in the form of a system of parallel thin metal plates, the planes of which are parallel to the direction of wave propagation and perpendicular to the vector electric field [23]. The plates of such a shield are located at a distance from each other less than half the wavelength of the operating frequency range. The disadvantage of the considered screens is that the radiation frequency increases with leakage of part of the power through it, which reduces its reflection coefficient. When it is used as a reflector of reflector antennas, the efficiency decreases, and the directional diagram of the antennas is distorted. No control reflection maxima due to changes in the thickness of the lattice elements. The proposed reflective screen contributes to an increase in the reflection coefficient in a wide frequency range, it is possible to control the maxima reflections due to changes in geometric characteristics, as well as an increasing inefficiency. The technical result is achieved by changing the design of the lattice, each lattice element is a multilayer hierarchically constructed self-similar metal-semiconductor composite and viewed as a fractal formation.

\section{Materials and Methods}

To simulate the electrical properties of the research object, we use the effective medium theory [24]. The essence of the effective medium model is that the system of clusters forming the composite material is considered as a kind of new medium with the same level of polarization. Thus, knowing the parameters of each of the components of the composite, their geometric shape and concentration, it is possible to determine the characteristics of the resulting composite medium as a whole. The advantage of this approach is that in order to analyze the propagation of an electromagnetic field in a composite medium, there is no need to solve Maxwell's equations at each point in space. The construction and analysis of such models are based on the solution of the electrostatics problem in a local field in a ball.

Let there be a ball with a dielectric constant $\varepsilon_{1}$, which is surrounded by a dielectric medium with permittivity $\varepsilon_{2}$. The local field $\bar{E}_{1}$ inside the ball is defined as the sum of the external field $\bar{E}_{0}$ and the Lorentz field $\bar{E}_{d}=-\left(\frac{4 \pi}{3}\right) \bar{p}$, the field of the polarized sphere. The 
direction of $\bar{E}_{d}$ depends on the ratio of $\varepsilon_{1}$ и $\varepsilon_{2}: \bar{E}_{1}=\bar{E}_{0}+\bar{E}_{d}=\bar{E}_{0}-\frac{4 \pi}{3} \bar{p}=\frac{3 \varepsilon_{2}}{\varepsilon_{1}+2 \varepsilon_{2}} \bar{E}_{0}$, where $\bar{p}=\chi_{0} \bar{E}_{0}$ is the vector the polarization of the medium in the ball, and $\chi_{0}$ is the polarizability of the sphere. Consider a medium of volume $\mathrm{V}$, with a dielectric constant $\varepsilon_{2}$, filled with spherical inclusions with a dielectric constant $\varepsilon_{1}$. The polarization of such a substance is determined by the sum of the polarization of the spherical inclusions: $\chi=\frac{1}{V} \sum_{i} V_{i} \chi_{0}$, where $V_{i}$ is the volume of the $i$-th particle, and $f_{1}=\frac{1}{V} \sum_{i} V_{i}$, volumetric filling factor. The volume is characterized by the so-called effective dielectric constant $\varepsilon_{e f f}$. Consider a composite medium consisting of a matrix and inclusions of spherical and cylindrical shapes of several types. Depending on the type, the shape of inclusions in the matrix of the composite, and the direction of the field action, within the framework of the effective medium theory, various models are given for determining the electrodynamic properties of the composite medium:

- Chaotically located spherical inclusions in the matrix [25]:

$$
\varepsilon_{e f f}=\varepsilon_{2}\left[1+v\left(\varepsilon_{1}-\varepsilon_{2}\right) /\left(\varepsilon_{2}+\frac{1-v}{3}\left(\varepsilon_{1}-\varepsilon_{2}\right)\right)\right] .
$$

- An ordered cubic system of spherical inclusions in a matrix [26]:

$$
\varepsilon_{e f f}=\varepsilon_{2}\left[1+3 v /\left(\frac{\varepsilon_{1}+2 \varepsilon_{2}}{\varepsilon_{1}-\varepsilon_{2}}-v-1.31 \frac{\varepsilon_{1}-\varepsilon_{2}}{\varepsilon_{1}+\frac{4}{3} \varepsilon_{2}} v^{3.33}\right)\right] .
$$

- Chaotically located cylindrical inclusions in the matrix under the influence of the field along the axes of the cylinders [27]:

$$
\varepsilon_{e f f}=\varepsilon_{1}\left[1+v\left(\varepsilon_{1}-\varepsilon_{2}\right) /\left(\varepsilon_{1}+\frac{1}{2}(1-v)\left(\varepsilon_{1}-\varepsilon_{2}\right)\right)\right] .
$$

- Chaotically located cylindrical inclusions in the matrix under the influence of a field perpendicularly directed relative to the axes of the cylinders [28]:

$$
\varepsilon_{e f f}=(v-0.5)\left(\varepsilon_{1}-\varepsilon_{2}\right)+\sqrt{\left((v-0.5)\left(\varepsilon_{1}-\varepsilon_{2}\right)\right)^{2}+\varepsilon_{1} \varepsilon_{2}} .
$$

On the basis of the above models, a software package was developed [29], which makes it possible to predict the electrodynamic characteristics of the composite depending on the type of inclusions, the frequency of external influence, which is indicated in the Equations (1)-(4) by $v$, and the quantitative ratio of the components that make up the composite. The tabular values of the parameters for titanium dioxide and aluminum oxide were taken as the initial data. Figures 1-3 show graphs of the dependences of the complex dielectric permittivity of $\mathrm{Si}, \mathrm{SiO}_{2}$, and $\mathrm{B}$ on the frequency of the external influence in the range of $0-80 \mathrm{MHz}$.

Figures 4 and 5 show the results of computer simulation of composite media consisting of a matrix and inclusions of various types. The following quantitative ratios of the components were considered: The volume fraction of the matrix -0.9 , the volume fraction of inclusions- -1 . 


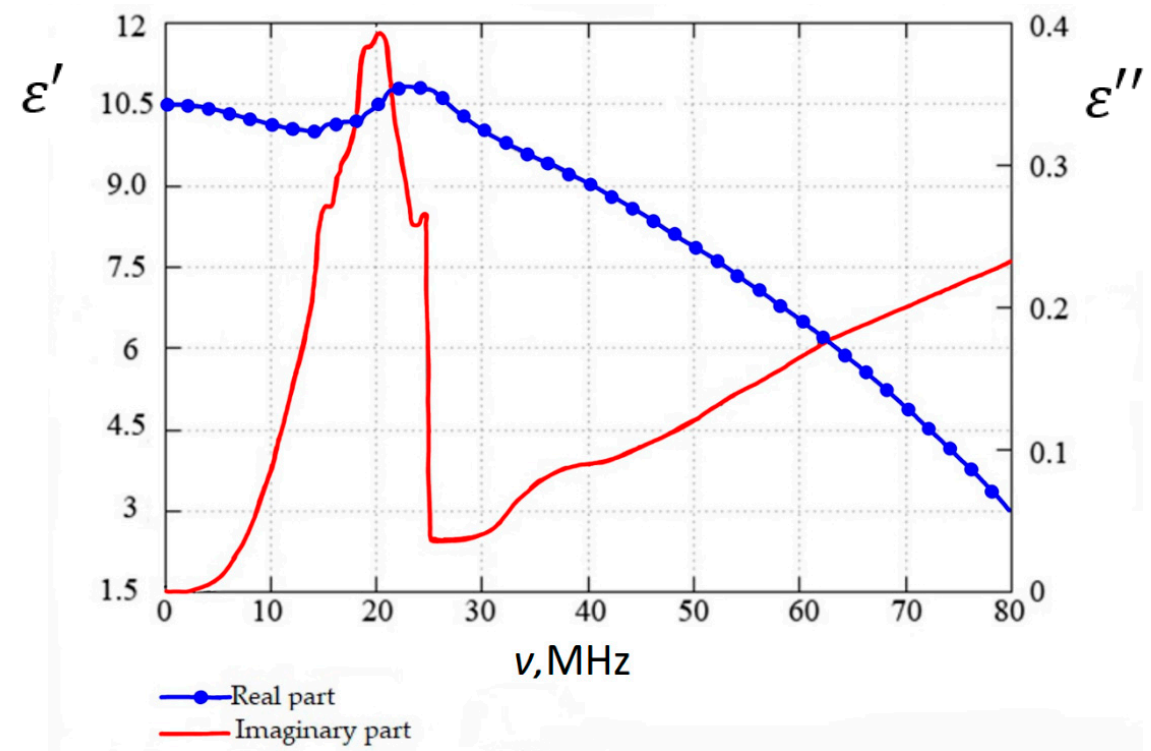

Figure 1. Frequency dependence of the complex dielectric constant of silicon.

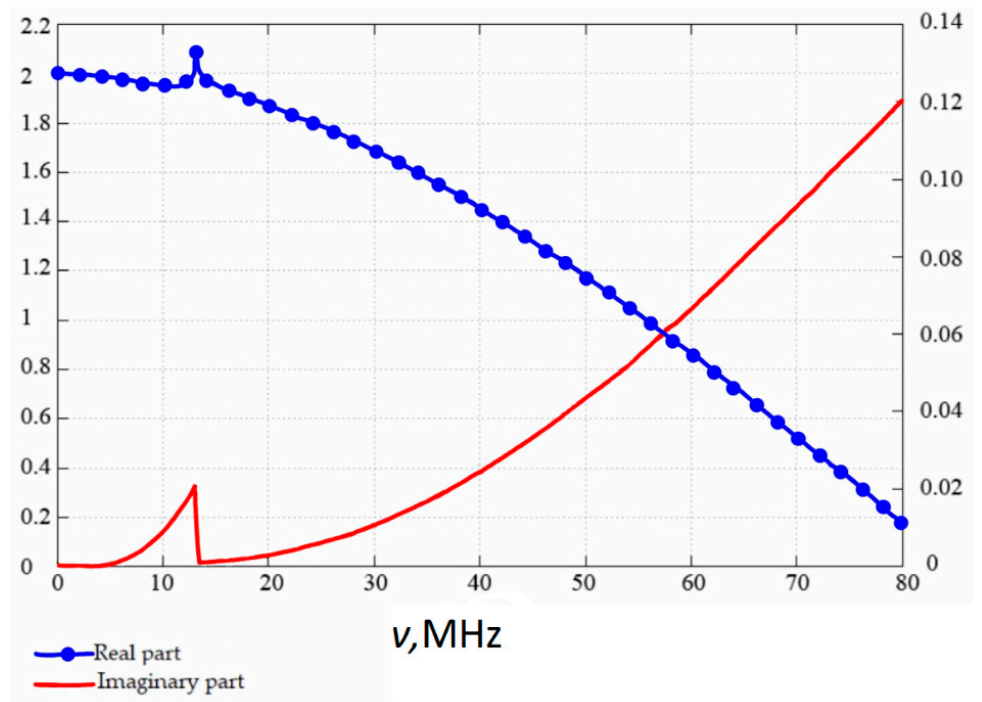

Figure 2. Frequency dependence of the complex permittivity of boron.

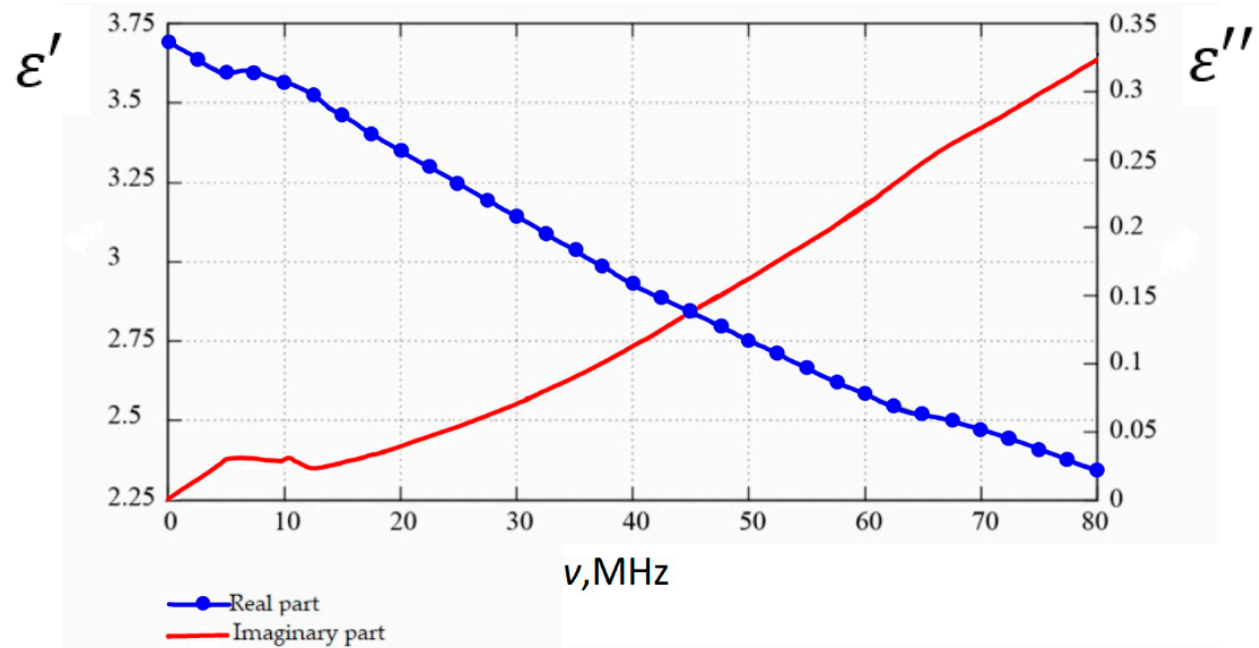

Figure 3. Frequency dependence of the complex dielectric constant of silicon dioxide. 


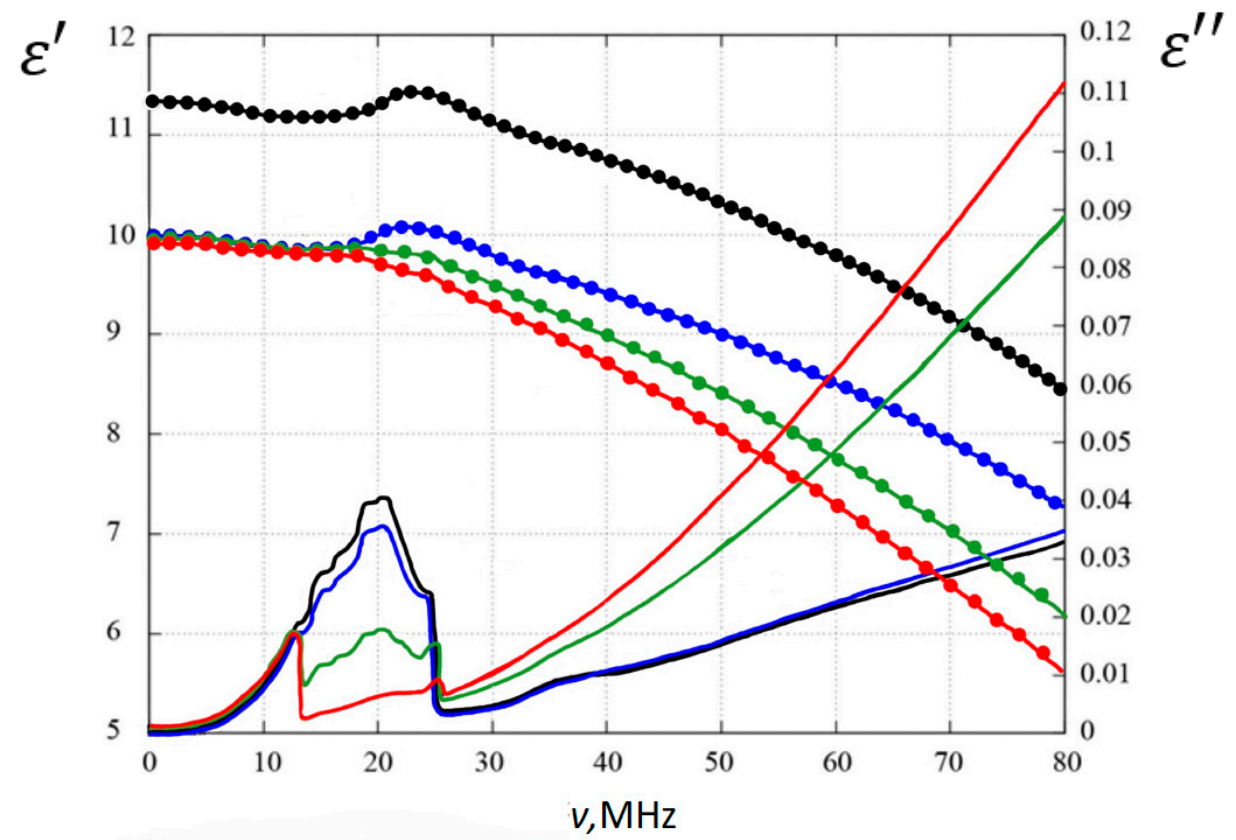

Chaotically located spherical inclusions $\bullet,-$

Ordered cubic system of spherical inclusions -

Randomly spaced cylinders:- - , - - field perpendicular to the axes;

$\sim,-$ - field parallel to the axes

Figure 4. Frequency dependences of the complex permittivity for cylindrical and spherical inclusions $B$ in a matrix $\mathrm{Si}$.

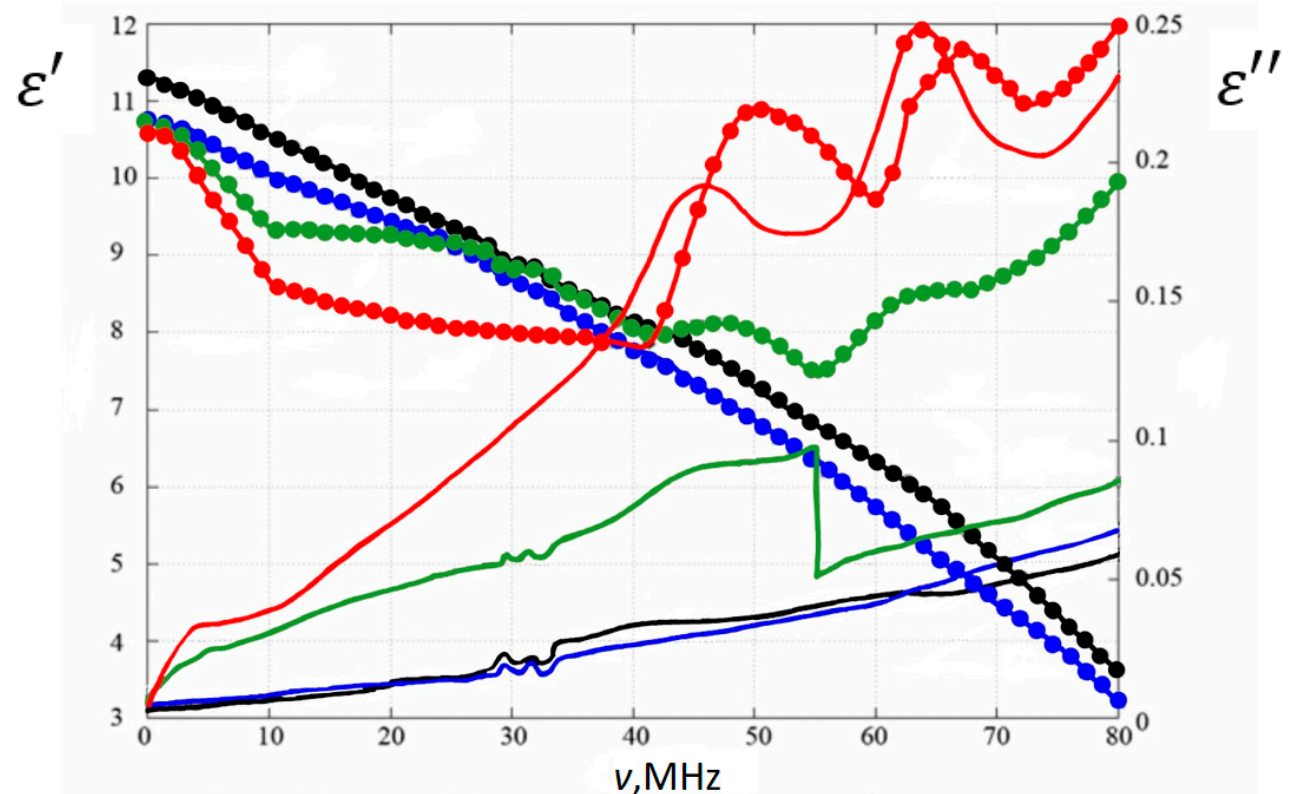

Chaotically located spherical inclusions $-\mathbf{-},-$

Ordered cubic system of spherical inclusions - - , -

Randomly spaced cylinders:- - - - - field perpendicular to the axes;

,$- \ldots$ - field parallel to the axes

Figure 5. Frequency dependences of the complex permittivity for cylindrical and spherical $\mathrm{SiO}_{2}$ inclusions in a matrix $\mathrm{Si}$.

\section{Results}

The work presents the developed mathematical model of the reflecting screen of fractal structure (Figure 6). The proposed structure of the screen makes it possible to increase 
the reflection coefficient in a wide frequency range with the possibility of controlling the reflection maxima by changing the geometric parameters of the structural elements.

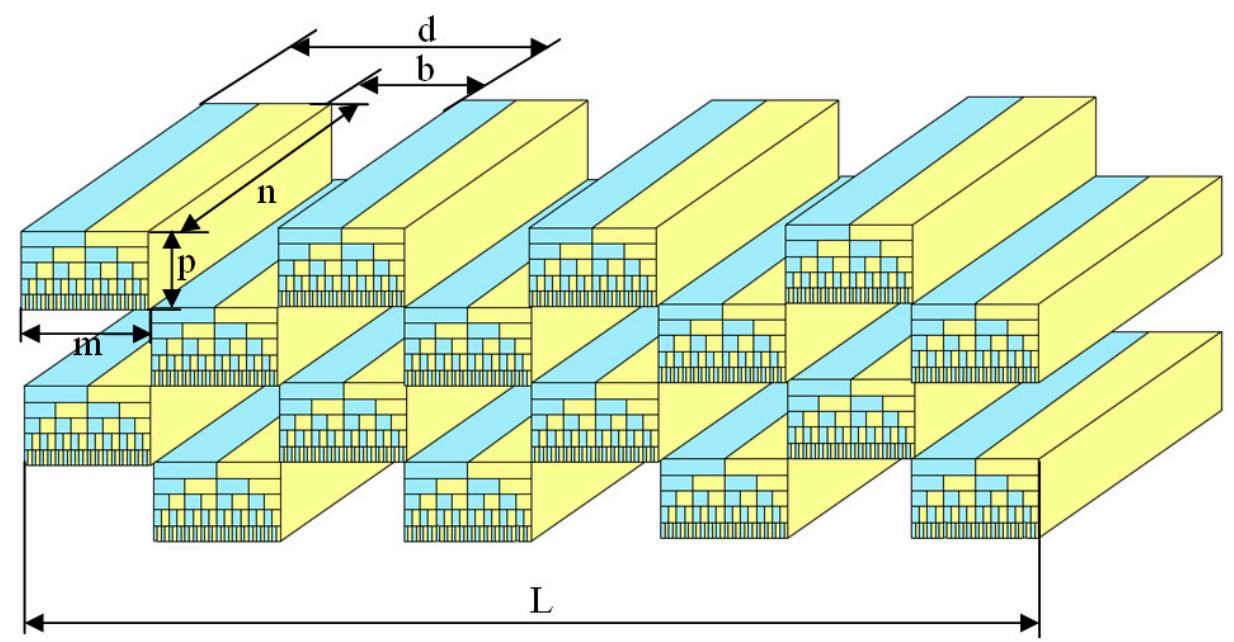

Figure 6. Reflective screen of fractal structure.

The period of the lattices, the distance between the lattices, the length and width of the lattice element are selected from the condition [30]:

$$
\cos \left(\frac{2 \pi d f_{\max }}{c}\right)+\frac{c}{2 b f_{\max } \ln \left(\frac{b}{\sqrt{m^{2}+n^{2}+p^{2}}}\right)} \sin \frac{2 \pi d f_{\max }}{c}=1 .
$$

where $b$-lattice period; $n, m, p$-length, width and height of a lattice element, $d$-distance between lattice elements, $f_{\max }$ - the maximum frequency of the operating frequency range of the screen, $c$-speed of light in vacuum, $L$-reflective screen length.

Each lattice element is a multilayer hierarchically constructed composite consisting of a metal and a semiconductor, which has a self-similar structure and is considered a fractal formation (Figure 7).

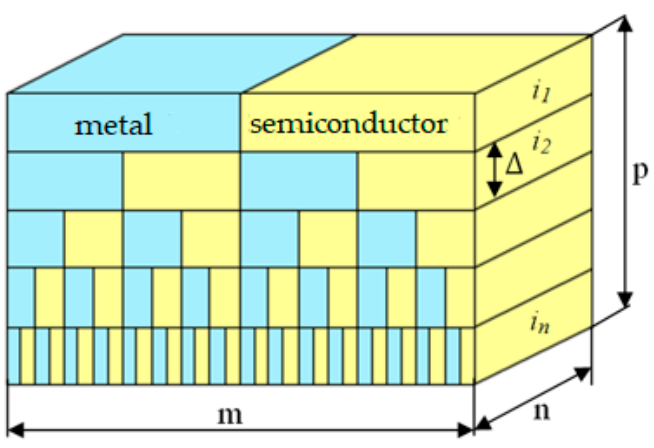

Figure 7. Reflective screen lattice element.

The minimum width of an element of a layer of a composite of a fractal structure can reach a size equal to the van der Waals radius of a chemical element of which the composite is composed.

The total number of lattice elements is chosen from the condition:

$$
m=\frac{W^{2}}{4 F d}
$$

where $W$-lattice width, $F$-reflector's focus. 
The calculation of the reflection coefficient $R$ of the screen was carried out as follows. The values of the reflection coefficient $R$ for a semi-infinite medium are determined from the relation given in [31]:

$$
R=\frac{(N-1)^{2}+\chi^{2}}{(N+1)^{2}+\chi^{2}}
$$

where $n$ and $\chi$-refractive and absorption indices of the medium, respectively.

The refractive and absorption indices included in Expression (1), as shown by the electrodynamic calculation, satisfy the following relations $[30,31]$ :

$$
\begin{aligned}
& \chi=\frac{c}{2 \pi d f} \operatorname{arcosh}\left[\cos \frac{2 \pi d f}{c}+\frac{c}{2 b f \cdot \ln \left[\frac{b}{\sqrt{m^{2}+n^{2}}}\right]} \sin \frac{2 \pi d f}{c}\right] \\
& N=\frac{c}{2 \pi d f} \arccos \left[\cos \frac{2 \pi d f}{c}+\frac{c}{2 b f \cdot \ln \left[\frac{b}{\sqrt{m^{2}+n^{2}}}\right]} \sin \frac{2 \pi d f}{c}\right]
\end{aligned}
$$

where $f$-frequency of the operating frequency range of the screen, $b$-lattice period, $d$-distance between lattice elements, $n, m$-length and width of a lattice element.

Figure 8 shows a graph of the dependence of the reflection index on the frequency of the wave of external electromagnetic radiation and the level of fractality of the lattice element $i(1: i=10,2: i=6,3: i=3)$, corresponding to the following parameters of the reflective screen

$$
\begin{aligned}
& 1-b=d=0.5 \mathrm{~cm} ; m=n=p=0.5 \mathrm{~cm} ; \\
& 2-b=d=0.5 \mathrm{~cm} ; m=n=p=0.045 \mathrm{~cm} ; \\
& 3-b=d=0.5 \mathrm{~cm} ; m=n=p=10^{-2} \mathrm{~cm} ; \\
& P=i \Delta .
\end{aligned}
$$

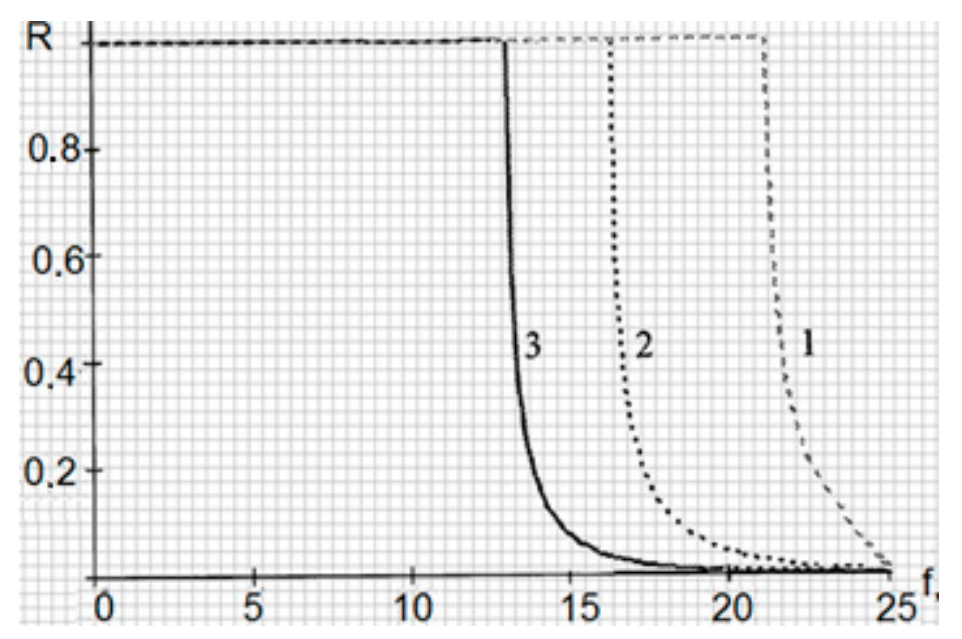

Figure 8. The graph of the dependence of the reflection index on the frequency of external electromagnetic radiation and the level of fractality of the lattice element.

The proposed device works as follows. Radiation falls on a reflecting screen. With the specified parameters, the reflection coefficient $R \geq 0.99999$ for $f \leq f_{\max }$.

As can be seen from Figure 8, the operating frequency range depends on the level of fractality of the lattice element of the reflecting screen. The fractal structure of the elements of the reflecting screen allows an increase in the operating frequency range by an average of $5 \mathrm{GHz}$ in comparison with the known reflecting screens with a traditional grating structure. By changing the level of fractality, it becomes possible to control the maxima of the reflection coefficient. 


\section{Conclusions}

In this work, the frequency dependences of the real and imaginary components of the effective dielectric constant of composite media consisting of a matrix and inclusions of spherical and cylindrical shapes are obtained. The dependences of the complex dielectric constant on the frequency of the external action of the electromagnetic field in the range of $1-80 \mathrm{MHz}$ of the composite of the following types of inclusions

- chaotically located spherical inclusions in the matrix;

- $\quad$ ordered cubic system of spherical inclusions in the matrix;

- randomly located cylindrical inclusions in the matrix under the influence of the field along the axes of the cylinders;

- close-packed cylindrical inclusions in the matrix under the influence of a field perpendicularly directed relative to the axes of the cylinders;

- randomly located cylindrical inclusions in the matrix under the influence of a field perpendicular to the axes of the cylinders.

The results obtained indicate that the structural features of the composite have a significant effect on the electrodynamic properties of the object under study. As shown by the type of inclusions, electrical layers on the boundary surfaces of dispersed particles significantly affect the complex dielectric constant.

Moreover, a model of a reflecting screen of a fractal structure was proposed in the work. If the proposed reflective screen is made with the parameters described in the article, then its efficiency, in comparison with a reflective screen with a traditional grating structure, will be increased by $5.5 \%$

In the future, the authors plan to continue research by conducting a full-scale experiment for the described model of the screen, as well as to continue modeling the reflecting screens of the fractal structure of other modifications.

Author Contributions: Data curation, D.S.; Formal analysis, V.B.; Investigation, P.N.; Validation, Y.Y.; Writing —original draft, S.K.; Writing—review \& editing, E.R. All authors have read and agreed to the published version of the manuscript.

Funding: No external funding.

Institutional Review Board Statement: Not applicable.

Informed Consent Statement: Not applicable.

Data Availability Statement: Study did not report any data.

Conflicts of Interest: The authors declare no conflict of interest.

\section{References}

1. Krakhmalev, O.; Krakhmalev, N.; Gataullin, S.; Makarenko, I.; Nikitin, P.; Serdechnyy, D.; Liang, K.; Korchagin, S. Mathematics model for 6-DOF joints manipulation robots. Mathematics 2021, 9, 2828. [CrossRef]

2. Terin, D.; Kardash, M.; Korchagin, S.; Tsyplyayev, S.; Cherkasov, V.; Druzhinina, T. Features of thermomechanical stability of anionic-cation exchange matrix "polikon ac" on viscose non-woven materials. Membranes 2021, 11, 734. [CrossRef] [PubMed]

3. Tretyakov, Y.D. Ceramics in the past, present, and future. Sorovsk Educ. J. 1998, 6, 53-59.

4. Travkin, P.G.; Vorontsova, N.V.; Vysotsky, S.A.; Lenshin, A.S.; Spivak, Y.M.; Moshnikov, V.A. Investigation of the regularities of the formation of the structure of porous silicon in multistage modes of electrochemical etching. Izv. SPbGETU LETI 2011, 4, 3-5.

5. Milosev, I.; Kapun, B. He corrosion resistance of Nitinol alloy in simulate physiological solutions: Part 1: The effect of surface preparation. Mater. Sci. Eng. 2012, 32, 1087-1090. [CrossRef]

6. Buyakova, S.P.; Khlusov, I.A.; Kulkov, S.N. Porous zirconium ceramics for bone endoprosthetics. Phys. Mesomech. 2004, 7, 127-130.

7. Aseev, A.L.; Popov, V.P.; Volodin, V.P.; Maryutin, V.N. Prospects for the Use of Silicon-on-Insulator Structures in Micro-Nanoelectronics and Microsystem Technology. Nano- and Microsystem Technology; New Technologies: Moscow, Russia, 2002; pp. $23-29$.

8. Nikitin, A.S. Prospects for the use of composite materials. Econ. Life 2012, 4, 6.

9. Kim, R.P.; Korchagin, S.A. Mathematical and computer simulation of the electrophysical properties of a multicellular structure exposed to nanosecond electrical pulses. Izv. Saratov Univ. New Ser. Ser. Math. Mech. Inform. 2021, 21, 259-266. [CrossRef] 
10. Korchagin, S.A.; Klinaev, Y.V.; Serdechnyy, D.V.; Terin, D.V. Software and Digital Methods in the Natural Experiment for the Research of Dielectric Permeability of Nanocomposites. In Proceedings of the International Conference on Actual Problems of Electron Devices Engineering, APEDE 2018, Saratov, Russia, 27-28 September 2018; pp. 262-265.

11. Korchagin, S.; Romanova, E.; Serdechnyy, D.; Nikitin, P.; Dolgov, V.; Feklin, V. Mathematical modeling of layered nanocomposite of fractal structure. Mathematics 2021, 9, 1541. [CrossRef]

12. Werner, D.H. An overview of fractal antenna engineering research. IEEE Antennas Propag. Mag. 2003, 45, 38-57. [CrossRef]

13. Pavlik, R. Cyclostationary and correlation based signal detection for harmonic radar systems. In Proceedings of the 2016 International Symposium on Fundamentals of Electrical Engineering (ISFEE), Bucharest, Romania, 30 June-2 July 2016 ; pp. 1-5.

14. Korchagin, S.A.; Klinaev, Y.V.; Terin, D.V.; Romanchuk, S.P. Modeling the electrodynamic properties of composite media. Bull. Saratov State Tech. Univ. 2015, 3, 48-54.

15. Pishkinas, A.O.; Onosov, I.A.; Korchagin, S.A. [and others] Development of software for modeling composite nanomaterials. Mod. Inf. Technol. IT Educ. 2015, 2, 301-305.

16. Bennett, R. Magnetic Coupling Device with Reflective Plate and Methods for Use Therewith. U.S. Patent Application No. 15/913,013, 6 March 2018.

17. Soomro, S.R.; Urey, H. Design, fabrication and characterization of transparent retro-reflective screen. Opt. Express 2016, 24, 24232-24241. [CrossRef] [PubMed]

18. Assis, A.N. Spontaneous emulsification of a metal drop immersed in slag due to dephosphorization: Surface area quantification. Metall. Mater. Trans. B 2015, 46, 568-576. [CrossRef]

19. Klein, R. Vital through Self-Concept Bias Modification? Using a Computer Based Intervention for Gaining Implicit and Explicit Vitality. Bachelor's Thesis, University of Twente, Enschede, The Netherlands, 2017.

20. Todorov, R.; Lozanova, V.; Knotek, P.; Černošková, E.; Vlček, M. Microstructure and ellipsometric modelling of the optical properties of very thin silver films for application in plasmonics. Thin Solid Film. 2017, 628, 22-30. [CrossRef]

21. Xie, Y.; Wang, L.; Liu, B.; Zhu, L.; Shi, S.; Wang, X. Flexible, controllable, and high-strength near-infrared reflective $\mathrm{Y}_{2} \mathrm{O}_{3}$ nanofiber membrane by electrospinning a polyacetylacetone-yttrium precursor. Mater. Des. 2018, 160, 918-925. [CrossRef]

22. Li, P.; Hu, G.; Dolado, I.; Tymchenko, M.; Qiu, C.-W.; Alfaro-Mozaz, F.J.; Casanova, F.; Hueso, L.E.; Liu, S.; Edgar, J.H.; et al. Collective near-field coupling and nonlocal phenomena in infrared-phononic metasurfaces for nano-light canalization. Nat. Commun. 2020, 11, 1-8. [CrossRef] [PubMed]

23. Wu, J.; Wu, F.; Lv, K.; Guo, Z.; Jiang, H.; Sun, Y.; Li, Y.; Chen, H. Giant Goos-Hänchen shift with a high reflectance assisted by interface states in photonic heterostructures. Phys. Rev. A 2020, 101, 053838. [CrossRef]

24. Vinogradov, A.P. Electrodynamics of Composite Materials; Editorial URSS: Moscow, Russia, 2001; p. 208.

25. Romanchuk, S.P.; Terin, D.V.; Kats, A.M.; Klinaev, Y.V. Mathematical modeling of structures and processes of interaction of electromagnetic radiation with CORE-SHELL nanoobjects. Bull. SSTU 2011, 4, 98-102.

26. Maxwell-Garnett, J.C. Colours in metal glasses and in metallic films. Philos. Trans. R. Soc. Lond. Ser. A Contain. Pap. A Math. Or Phys. Character 1904, 203, 385-420.

27. Netushil, A.V.; Zhukovitskiy, B.Y.; Kudin, V.N.; Parini, E.P. High-Frequency Heating of Dielectrics and Semiconductors; Gosenergoizdat: Moscow, Russia, 1958; pp. 103-119.

28. Sherman, P. Emulsion Science; Academic Press: London, UK; New York, NY, USA, 1968; p. 329.

29. Romanchuk, S.P.; Terin, D.V. Mathematical Modeling and Multicriteria Analysis of Nonlinear Properties of Composite Materials Based on an Effective Medium. Certificate of State Registration of Computer Programs No. 2014612918/69. Available online: https: / / www.fips.ru/iiss/document.xhtml?faces-redirect=true\&id=62fb4a644e04dac53dc4964538462d92 (accessed on 8 December 2021).

30. Korchagin, S.A.; Terin, D.V. Reflecting Screen of Fractal Structure “FRAKSTRON". Utility Model Patent RU 178702 U1, 17 April 2018; Application No. 2017119689, 6 May 2017. Available online: https://www.elibrary.ru/download/elibrary_38152498_91850 564.PDF (accessed on 1 December 2021).

31. Born, M. Fundamentals of Optics, 2nd ed.; The Science: Moscow, Russia, 1973; p. 720. 